\title{
SPECTRAL ESTIMATION OF SHORT SEGMENTS OF EEG SIGNALS USING LEAST SQUARES WAVESHAPING FILTERS
}

\author{
Rajamma Mathew and D. Narayana Dutt \\ Department of Electrical Communication Engg. \\ Indian Institute of Science \\ Bangalore-560 012, INDIA
}

\begin{abstract}
Electroencephalogram (EEGJ, the manifestations of brain's electrical activity as recorded on the scalp, has become an indispensable tool in clinical neurophysiology and related fields. The main objective of EEG signal analysis is to extract valid information from EEG. records and for this purpose many analysis techniques have been used. As far as background activity is concerned, spectral analysis is important to determine the rhythms present. Often short segrnents of data is to be analysed in order to adequately characterise the temporally rapid changes that occur in EEG. This paper is concerned with this aspect of EEG analysis viz., spectral estimation of short segment EEG data. Autoregressive (AR) spectral es imation techniques are known to provide better resolution than classical periodogram methods when short segments of data are selected for analysis. We suggest in this paper a method which works better than the normal AR spectral estimation for very short segments of data. It has been observed that the energy in the EEG data segment is concentrated not in the beginning but somewhere in between the initial and the final positions thus confirming ti e fact that EEG is a mixed delay signal. This position where the energy is concentrated has been obtained with the help of least squares waveshaping filter. It is also shown that the knowledge of the position where the energy in the signal is concentrated can be used in making a better spectral estimation of short segments of EEG data. The study has been made first with the simulated data and then with the actual EEG data. It is also observed that the performance of the present method and AR method become comparable as the length of data segment increases.
\end{abstract}

\section{Introduction}

The spontaneous electrical activity of the brain is normally measured by means of electrodes placed on the scalp of the subject. The recordings known as electroencephalogram (EEG), show certain periodicities or sharp resonances accompanied by randomness. These signals carry information regarding the medical status of the brain and are used by physicians as a means of diagnosis. It has long been observed that the frequency content of the
EEG signal is of crucial importance in its assessment. The rhythmic activity corresponding to frequency centered around $10 \mathrm{~Hz}$ and ranging from 8 to $13 \mathrm{~Hz}$ is called the alpha activity and the high frequency rhythmic activity from 14 to $30 \mathrm{~Hz}$ is called the beta activity. The low frequency activities in the range 0.5 to $3 \mathrm{~Hz}$ and 4 to $7 \mathrm{~Hz}$ are called the delta and theta activities respectively. The appearance of these activities depends on the location on the scalp, the state of the subject and various other factors. Electroencephalography has been found to be a useful tool for studying the functional states of the brain and also for diagnosing injuries and functional brain disturbances. Its use is well established in monitoring sleep, depth of anaesthesia and cessation of brain function [1]. Since last two decades, computer based signal processing techniques are known for their inherent desirable features like speed of operation, quantification and precise characterization of the signal, consistency and ability to do statistical manipulations in bringig out subtle results [2]. The present, availability of fast minicomputers at low cost has particularly enhanced their importance and utility.

EEG is often analysed as a time series like speech and seismic signals. The signal has to satisfy certain requirements to achieve valid results by the analysis method employed and this necessitates a knowledge about the characteristics of the EEG signal. EEG is a random signal and as such it needs to be treated as a stochastic process like signals in other fields mentioned above. The need for an understanding of a signal as complex as the EEG signal has led users, almost since the beginning, to search for means to extract the meaningful part of EEG and to analyse it. It has been observed that the spectral content of the background activity and the characteristics of the transients adequately represent the EEG record and hence processing is concerned with the extraction of the features of these two aspects. Techniques of EEG analysis broadly fall into two categories, viz., the time domain and the frequency domain. In general, frequency domain approach is suitable for analysis of background activity and the time domain techniques for paroxysmals (transients). However, time and frequency domain techniques are applied for both cases without much restriction

Frequency domain representation provides better insight into the generation of EEG signals, is more descriptive of pattern 
fluctuations and enables valid comparisons. It has gained popularity over time domain representation. Walter [3] first introduced digital spectral analysis to EEG and with the advent of FFT, the frequency domain analysis has become very popular in both experimental and clinical EEG. It has been observed, however, that the periodogram is not a consistent estimate of the spectrum as the stability does not improve with the increase in the number of samples. Other major problems associated with FFT method arc the leakage errors due to high side lobe levels of the window function applied to the time function and the poorer resolution. Also the spectral analysis does not provide any substantial data reduction and the data is only in different form. In order to overcome some of these limitations, parametric representation of EEG signals has been used [4]. In particular, the autoregressive (AR) model has been widely used for EEG analysis mainly to get better spectral resolution [5]. The studies of Rappelsberger and Petsche [6] indicate that the $\mathbf{A R}$ model provides better spectral estimates of the true spectrum than by the FFT method and the length of the data required is smaller than that required by the FFT method. The

- studies of Blinowska et al., [7]also show that AR model provides better resolution with much smaller statistical fluctuations than by FFT method.

For the study of features, EEG is usually devided into short segments. Short epochs are typically selected for analysis in preference to long ones in order to be able to adequately characterize the temporally rapid changes that occur in EEG. Correct estimation of these characteristics are of tantamount importance since the remaining analysis is based upon it. It has already been mentioned that the resolution of the resulting spectra obtained by applying Fourier transform to short segments of data is very poor and that better resolution can be obtained by applying AR modelling. It is generally found that $\mathbf{A R}$ model works very well for short segments of data of duration around one second. In this paper, we suggest a method which works better than the normal AR spectral estimation for still shorter segments of data (data segments of length 0.5 seconds and below).

\section{Theorv}

Waveshaping filters are a class of digital filters which shapes a given input signal into a desired output signal. In the special case when the desired signal is a unit impulse, they are known as spiking filters. Spiking filters can be used to determine the position where the energy in the signal is mostly concentrated. In this paper, the significance of estimating this position of energy concentration in the spectral analysis of EEG signals is explored. A method which works better than the normal AR spectral estimation method for very short segments of data is suggested.

A signal is said to be minimum phase, if its Fourier transform is of minimum phase lag. Similarly a signal is said to be maxi- mum phase if its Fourier transform is of maximum phase lag. For a minimum phase sequence, all its poles and zeros will lie inside the . unit circle, whereas for a maximum phase sequence all its poles and zeros will lie outside the unit circle. Signals which are not minimum or maximum phase are known as mixed phase signals. Minimum phase signals are also known as minimum delay signals because, for all the sequences having the same Fourier transform magnitude, minimum phase sequence is delayed the least. In an analogous manner maximum phase sequence is delayed the most and mixed phase sequences have delays in between [8].

Another way to observe the above is that sequences that have the same Fourier transform magnitude have the same total energy (by Parseval's theorem). However, the energy in the minimum phase sequence will be concentrated around $n=0$ (beginning), whereas the energy in the maximum phase sequence will be concentrated around the final point. For a mixed phase sequence, the energy will be concentrated in between the initial and final points. Studies have already shown that EEG is a mixed delay signal. In order to find out the position where the energy in the signal is concentrated, waveshaping filters can be used. The problem may be formulated as follows (9):

Given a record of available data $\mathbf{I}$,

$$
\left[\begin{array}{lllll}
x_{0} & x_{1} & x_{2} & \cdots & x_{N}
\end{array}\right]
$$

find the best linear FIR filter with unit sample response sequence $h_{n}$ of order $M$

$$
\left[\begin{array}{lllll}
h_{0} & h_{1} & h_{2} & \cdots & h_{M}
\end{array}\right]
$$

which reshapes $x_{n}$ into the desired signal $y_{n}$

$$
\left[\begin{array}{lllll}
y_{0} & y_{1} & y_{2} & \cdots & y_{N+M}
\end{array}\right]
$$

The estimate of output of the above waveshaping filter will be

$$
\hat{y}_{n}=\sum_{m=0}^{M} h_{m} x_{n-m} \quad 0 \leq n \leq N+M
$$

The estimation error is

$$
e_{n}=y_{n}-\hat{y}_{n} \quad 0 \leq n \leq N+M
$$

As the optimality criterion, we choose the least squares criterion. We minimize $E$, which is given by

$$
E=\sum_{n=0}^{N+M} e_{n}^{2}
$$

The optimal filter weights $h_{m}$ are selected to minimize $E$. Wedefinethe $(N+M+1) \times(M+1)$ data matrix $\mathrm{X}$, the $(M+1) \times 1$ vector of filter weights $h_{\text {, the }}(N+M+1) \times 1$ vector of desired samples $\mathbf{y}$, and the estimation errors e, as follows: 


$$
\begin{aligned}
\mathbf{X} & =\left|\begin{array}{cccll}
x_{0} & 0 & 0 & \cdots & 0 \\
x_{:} & x_{0} & 0 & \cdots & 0 \\
x_{2} & x_{1} & x_{0} & \cdots & 0 \\
\cdot & \cdot & \cdot & \cdots & \cdot \\
\cdot & \cdot & \cdot & \cdots & \cdot \\
x_{N} & x_{N-1} & \cdot & \cdots & x_{N-M} \\
0 & x_{N} & \cdot & \cdots & \cdot \\
0 & 0 & x_{N} & \cdots & \cdot \\
\cdot & \cdot & \cdot & \cdots & \cdot \\
\cdot & \cdot & \cdot & \cdots & \cdot \\
\cdot & \cdot & \cdot & \cdots & x_{N}
\end{array}\right| \\
\mathbf{h} & =\left[\begin{array}{lllll}
h_{0} & h_{1} & h_{2} & \cdots & h_{M}
\end{array}\right]^{T} \\
\mathbf{y} & =\left[\begin{array}{lllll}
y_{0} & y_{1} & y_{2} & \cdots & y_{N+M}
\end{array}\right]^{T}
\end{aligned}
$$

where superscript $T$ represents the transpose of the matrix. From above we can write

$$
\hat{\mathbf{y}}=\mathrm{Xh} ; \quad \mathrm{e}=\mathbf{y}-\hat{\mathrm{y}} ; \quad E=\mathrm{e}^{T} \mathrm{e}
$$

Minimizing $E$ with respect to weight vector $\mathbf{h}$ results in orthogonality equations

$$
\mathrm{X}^{T} \mathrm{e}=\mathrm{X}^{T}(\mathrm{y}-\mathrm{Xh})=0
$$

which are equivalent to normal equations

$$
\mathbf{X}^{T} \mathbf{X h}=\mathbf{X}^{T} \mathbf{y}
$$

Solving for $\mathbf{h}$ we find

$$
\mathbf{h}=\left(\mathbf{X}^{T} \mathbf{X}\right)^{-1} \mathbf{X}^{T} \mathbf{y}=\mathbf{R}^{-1} \mathbf{r}
$$

where $\mathbf{R}=\mathbf{X}^{T} \mathbf{X}$ and $\mathbf{r}=\mathbf{X}^{T} \mathbf{y}$ are autocorrelation and crosscorrelation matrices respectively.

The actual output of the filter. $\hat{\mathbf{y}}$ is expressed as

$$
\hat{\mathbf{y}}=\mathbf{X h}=\mathbf{X R}^{-1} \mathbf{X}^{T} \mathbf{y}=\mathbf{P y}
$$

where $\mathbf{P}=\mathbf{X}\left(\mathbf{X}^{T} \mathbf{X}\right)^{-1} \mathbf{X}^{T}$

The error vector

$$
\mathbf{e}=(\mathbf{I}-\mathbf{P}) \mathbf{y}
$$

where $\mathbf{I}$ is the identity matrix and $\mathbf{P}$ is the performance matrix.

Since the per:ormance matrix $\mathbf{P}$ is a projection matrix, (I-P) is also a projertion matrix. Then the error squared becomes

$$
E=\mathbf{e}^{T} \mathbf{e}=\mathbf{y}^{T}(I-\mathbf{P})^{2} \mathbf{y}=\mathbf{y}^{T}(I-P) \mathbf{y}
$$

The $(\dot{M}+N+1) \times(M+N+1)$ matrix $\mathbf{P}$ has a trace equal to $M+1$. Since its eigenvalues as a projection matrix are either 0 or 1 , there must be $M+1$ eigenvalues that are equal to 1 and $N$ eigenvalues equal to zero. Therefore the matrix $P$ has a rank $M+1$, and if the desired vect ir $y$ is selected to be any of the
$M+1$ eigenvectors belonging to eigenvalue 1 , the corresponaing estimation error will be zero.

Among all possible waveshapes for $\mathbf{y}$, the most important ones are spikes or impulses. In this case, the waveshaping filter is also known as the spiking filter. Here $\mathbf{y}$ is an impulse at a delay equal to $i$, and can be represented as

$$
\begin{gathered}
\mathbf{y}=\mathbf{u}_{i}=\left[\begin{array}{llllll}
0 & \cdots & 0 & 1 & 0 & \cdots
\end{array}\right] \\
i^{\text {th }} \text { slot }
\end{gathered}
$$

where $0<i<N+M$

The actual output of the $i^{\text {th }}$ spiking filter is

$$
\hat{\mathbf{y}}=\mathbf{P y}=\mathbf{P u}, \quad \text { the } i^{\text {th }} \text { column of } \mathbf{P}
$$

Therefore the $i^{t h}$ column of the matrix $\mathbf{P}$ is the output of the $i^{t h}$ spiking filter which attempts to compress $x_{n}$ into a spike with $i$ delays. The corresponding $i^{\text {th }}$ filter is

$$
\mathrm{h}=\mathbf{R}^{-1} \mathbf{X}^{T} \mathbf{u}_{i}
$$

Therefore the columns of the matrix

$$
\mathbf{H}=\mathbf{R}^{-1} \mathbf{X}^{T}
$$

are all the optimal spiking filters. The estimation error of the $i^{\text {th }}$ spiking filter is

$$
E_{i}=\mathbf{u}_{i}^{T}(\mathbf{I}-\mathbf{P}) \mathbf{u}_{i}=1-\mathbf{P}_{i i}
$$

where $\mathbf{P}_{\boldsymbol{i i}}$ is the $i^{\text {th }}$ diagonal element of $\mathbf{P}$. Thus the optimum delay ( $i=n_{0}$ ) is selected as that column of $\mathbf{P}$, which has the maximum of $\mathbf{P}_{\boldsymbol{i i}}$. This optimum spiking delay gives the position around which the energy in the signal is concentrated.

\subsection{Spectral Estimation}

Any function $x(n)$ can be expressed as the convolution of a minimum phase function'and an all-pass function [10].

$$
x(n)=x_{1 \min }(n){ }^{*} x_{2 \text { ap }}(n)
$$

An AR filter tries to approximate $x_{1 \min }(n)$. It is found that if $x(n)$ itself is minimum phase, the approximation will be better. Any mixed phase function can be written as the convolution of a minimum phase function and a maximum phase function.

$$
x(n)=x_{1 \min }(n){ }^{*} x_{2 \max }(n)
$$

But we know that

$$
x_{2 \max }(n)=x_{2 \min }\left(n_{0}-n\right)
$$

where $n_{0}$ is the length of the maximum delay component. Therefore we can write in z-domain

$$
X(z)=X_{1 \min }(z) \cdot X_{2 \min }\left(z^{-1}\right) \cdot z^{-n_{0}}
$$


It is this delay $n_{0}$ that we are trying to find out using spiking filter. The knowledge of the spiking position $n_{0}$ give us an information of how much shift is to be given to make the original mixed phase signal to a near minimum phase signal. By approximating the resulting shifted signal by an AR model, we are able to get an improved estimate of the spectrum.

\section{Results and Discussion}

Application of spiking position theory to $\mathrm{EE}$ ? data showed that for most of the segments of EEG, the opt inum delay position is somewhere in between the initial and final positions confirming the fact that EEG is a mixed delay signal.

Fig. Ia and Fig. $1 b$ show the performance $\left(P_{i i}\right)$ of spiking filters plotted against the delay $i$, for two actual EEG data segments. It can be seen from the plots that the best performance is not at zero delay, but somewhere after that.

Our studies have shown that the knowledge of optimum spiking position can be used in spectral estimation efficiently. For very short segments of data, we advanced the signal by the amount of spiking delay and found the spectrum using linear prediction (LP) method. It is found that the spectrum of the shifted sequence gives better results than the LP spectrum of the original sequence.

Fig. 2 shows a comparison of spectra obtained by the two methods (curve ' $\mathbf{a}$ ' shows the spectrum of the shifted sequence and curve ' $b$ ' shows the spectrum of the original sequence) for simulated EEG data. The simulated data contains three sinusoids in white noise. The sinusoids have frequencies $10 \mathrm{~Hz}$ (relative amplitude 2), $14 \mathrm{~Hz}$ (relative amplitude 5) and $20 \mathrm{~Hz}$ (relative amplitude 0.5). The signal is sampled at $100 \mathrm{~Hz}$ and a data segment of 50 samples (corresponding to $0.5 \mathrm{sec}$. of data) is taken for the study. The optimum spiking delay position is found to be 15 . We advanced the data sequence by 15 samples and the LP spectrum is found out (number of AR parameters 12). It is seen in Fig.2 that the shifted sequence spectra gives better results than the spectrum of the original sequence.

Fig. 3 shows similar results for actual EEG data. An EEG segment of 64 samples (corresponding to $0.25 \mathrm{sec}$. of data) is taken. Visual observation of the data segment shows alpha activity predominantly, superimposed with power hum. The spiking delay position is found to be at 60 . Comparison of curves ' $a$ ' and ' $b$ ' in Fig. 3 shows that the new method gives better peaks.

\section{References}

[1] Gevins, A.S., Yeager, C.L., Diamond, S.L., Spire, J.P., Zeitlin, G.M, and Gevins, A.H., "Automated analysis of the electrical activity of the human brain (EEG): A progress report", Proc. IEEE, Vol.63, pp.1382-1399, 1975.

[2] Remand,-A and Storm Van Leeuwen, W., "Why analyse, quantify or process routine clinical EEG",
EEG Informatics: A didactic review, Ed. Remond, A., Elsevier/ North Holland, Amsterdam, pp.1- 8, 1977.

[3] Walter, D.O., "Spectral analysis of electroencephalogram: Mathematical determination of neurophysiological relationship from records of limited data", Experimental Neurology, Vol.8, pp.155-181, 1963.

[4] Isacksson, A., Wennberg, A. and Zetterberg, L.H., "Computer analysis of EEG signals with parametric models", Proc. IEEE, Vol.69, pp. 451-461, 1981.

[5] Fenwick, P.B.C., Mitchie, P., Dollimore, J. and Fenton, G.W., "Application of the autoregressive model to EEG analysis", Agressologic, Vol.10, pp 553, 1969.

[6] Rappelsberger, P. and Petsche, H., "Spectral analysis of the EEG by means of autoregression", In CEAN : Computerised EEG analysis, Eds. Dolce and Kunkel, Gurtav Fischer Verlag, Stuttgart, pp. 25-40, 1975.

[7] Blinowska, K.J., Czerwosz, L.T., Drabik, W., Pranarzczuk, P.J. and Ekiert, H., "EEG data reduction by autoregressive representation and discriminant analysis procedures", Electro. Clin. Neurophysiol., Vol. 51, pp. 650-658, 1981.

[8] Robinson, E.A., Statistical communication and detection Hafner, New York, 1967.

[9] Orfanidis, $\quad$ S.J., $\quad$ Optimum Signal Processing: An Introduction, McGraw Hill, New York, 1988.

[10] Oppenheim, A.V. and Schafer, R.W., Digital Signal Processing, Prentice Hall, Englewood Cliffc, N.J, 1975.

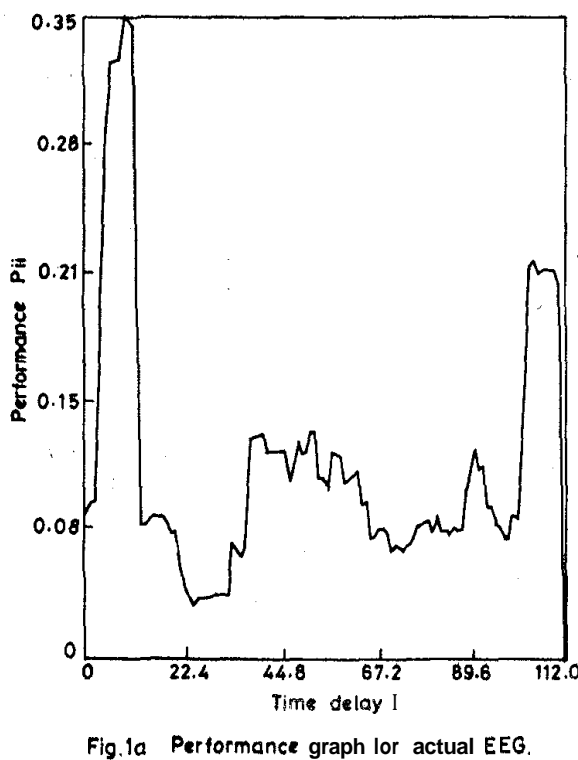




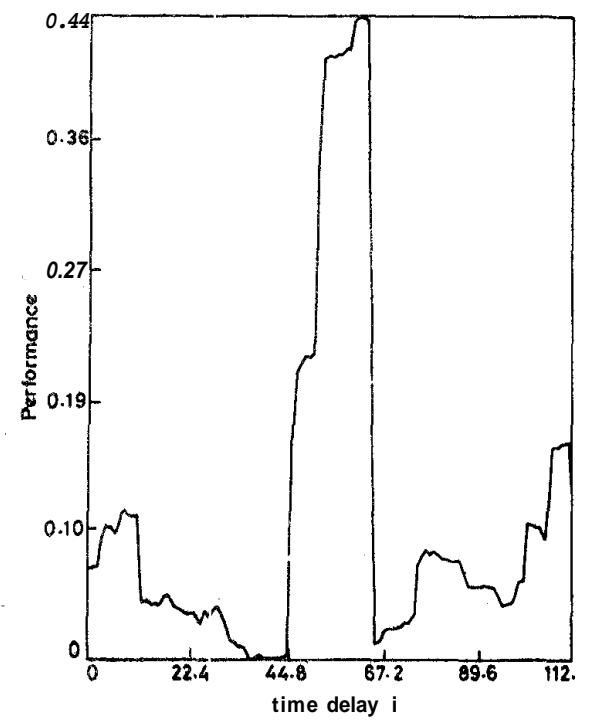

Fig. 16 Parrormance graph for actual EEG

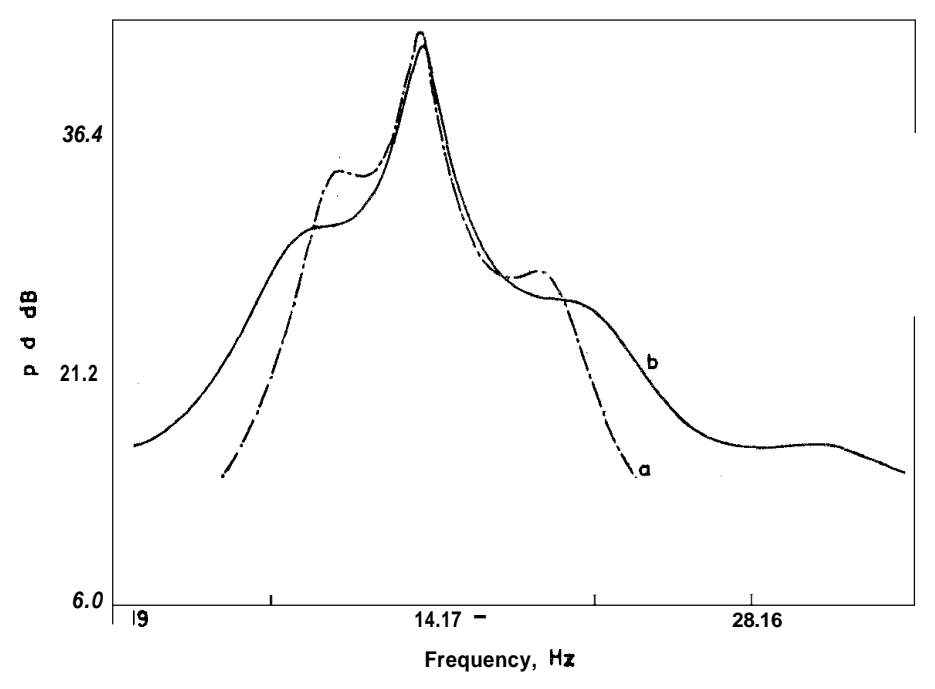

Fig. 2 Comparison of spectra for simulated data.

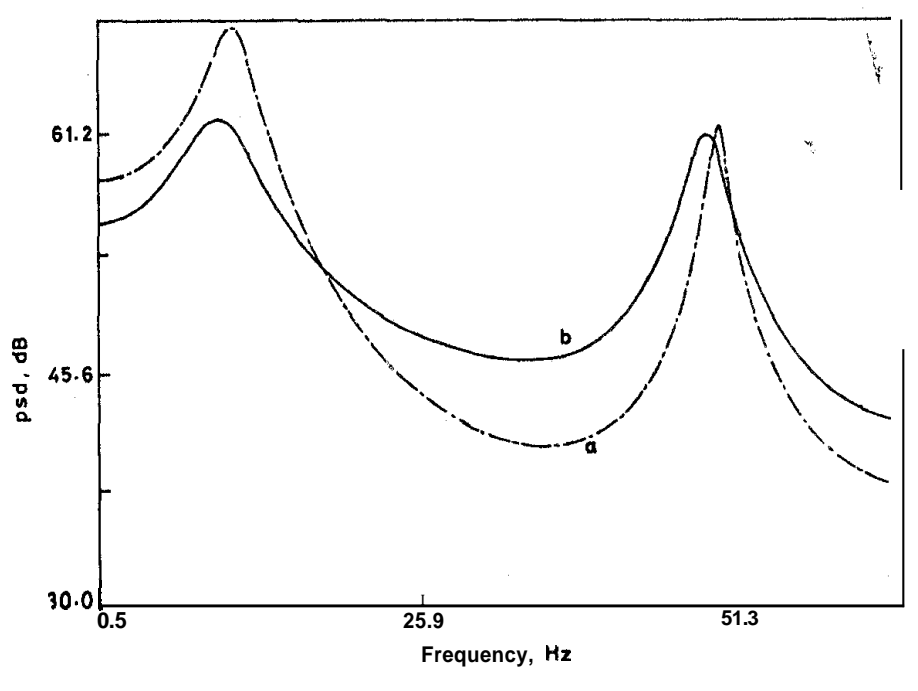

Fig 3 Comparison of spectra for actual EEG data 